\title{
LOS INICIOS DE LA CIENCIA MODERNA EN EL CARIBE NEOGRANADINO: PEDRO LÓPEZ DE LEÓN TEORÍA Y PRÁCTICA EN LA CIRUGÍA DE LA CARTAGENA DEL SIGLO XVII ${ }^{1}$
}

\author{
Antonino Vidal Ortega \\ Centro de Estudios Caribeños. Pontifica Universidad Católica Madre Maestra, \\ Santo Domingo. República Dominicana \\ Email: antoninovidal@pucmm.edu.do \\ ORCID iD: https://orcid.org/0000-0002-0012-325X \\ Jairo Solano Alonso \\ Universidad Simón Bolívar, Barranquilla, Colombia. \\ Email: jaisolano2003@yahoo.com \\ ORCID iD: https://orcid.org/0000-0001-7710-4682
}

Recibido: 1 octubre 2019; Aceptado: 13 abril 2020

Cómo citar este artículo/Citation: Vidal Ortega, Antonino; Solano Alonso, Jairo (2020) "Los inicios de la ciencia moderna en el Caribe Neogranadino: Pedro López de León teoría y práctica en la cirugía de la Cartagena del Siglo XVII”, Asclepio, $72(2)$ : p316. https://doi.org/10.3989/asclepio.2020.17

RESUMEN: La pregunta de quién fue el cirujano sevillano Pedro López de León y qué significó el manual de medicina y cirugía que escribió en Cartagena de Indias a comienzos del siglo XVII, es el objetivo central que persigue nuestro trabajo. A través de la reconstrucción de su vida, de las reflexiones en torno a su obra y de un análisis de los referentes bibliográficos que usó, resaltaremos la notoriedad trasatlántica que alcanzó en el esfuerzo por desarrollar el conocimiento científico de la medicina moderna en un puerto central del Caribe en el comercio Atlántico en la edad Moderna.

PALABRAS CLAVE: Historia del Caribe; Historia de la Medicina; Historia Colonial; Historia Moderna; Historia conectadas

THE BEGINNINGS OF MODERN SCIENCE IN THE NEOGRANADINO CARIBBEAN: PEDRO LÓPEZ DE LEÓN, THEORY AND PRACTICE OF SURGERY IN THE XVII CENTURY CARTAGENA DE INDIAS

ABSTRACT: The focus of this work is surgeon Pedro López de León and the contribution of his manual of medicine and surgery, which he wrote in Cartagena de Indias in the early seventeenth century. Through the reconstruction of his life, and the analysis of his work and bibliographical references, we highlight the transatlantic notoriety of the effort to develop the scientific knowledge of modern medicine in a central port of the Caribbean in the Atlantic Trade.

KEY WORDS: Caribbean History; History of Medicine; Colonial History; Modern History; Entangled History

Copyright: @ 2020 CSIC. Este es un artículo de acceso abierto distribuido bajo los términos de la licencia de uso y distribución Creative Commons Reconocimiento 4.0 Internacional (CC BY 4.0) 
La expedición botánica, impulsada por el científico andaluz Celestino Mutis, ha sido considerada por la historiografía iberoamericana la máxima empresa científica del periodo colonial, tal vez porque su contribución fue al tiempo educativa y científica. (Amaya, 1986. Frías, 1994. Hernández, 1982. Leal, 2018) Procesos modélicos de la llustración fueron las expediciones promovidas por la Corona Española en la segunda mitad del siglo XVIII, entre ellas la geodésica a las regiones equinocciales, adelantada por Jorge Juan y Antonio de Ulloa en compañía de La Condamine, Bougier y Goudín; (Jorge y Ulloa, 1748) las exploraciones de Humboldt y Bompland; (Von Humboldt, 1820) el inconcluso intento de Damián Churruca y José Joaquín Fidalgo de llevar a cabo el Atlas de América septentrional; (Fidalgo, 2011) los experimentos agrarios y educativos de José de Astigarraga en la remota gobernación de Santa Marta (Vidal, 2018)y la Expedición Botánica enmarcan un periodo de revolución del conocimiento y el uso regular de la estadística.

Con Celestino Mutis se formaron Francisco José de Caldas, que estimuló la física y las ciencias naturales en la Nueva Granada; Eloy Valenzuela, Francisco Antonio Zea; quien fuera director del Jardín Botánico de Madrid, José Tadeo Lozano, zoólogo, botánico y pedagogo; el pintor Salvador Rizo; y José Ignacio de Pombo, promotor de la ciencia en el caribe neogranadino; (Soto, 2004, 2005) el cartagenero Fray Diego García, explorador en busca de las especies granadinas de la Quina (Mantilla y Díaz, 1944) y Pedro Fermín de Vargas, exponente de los estudios en las áreas agropecuarias (Tisnés, 1979). Entre todos, ligados al magisterio de Mutis, sentaron las bases y precedentes de la ciencia moderna en Colombia.

Sin desconocer las razones que asisten a los impulsores de este enfoque dominante, aplicado incluso al estudio de los avances médicos, cuya modernización se asocia al reformismo borbónico y la acción de Mutis y Miguel de Isla como impulsores ideológicos de la renovación de la medicina (Quevedo, 2008), pensamos que es importante poner en valor los primeros tratados médicos y de cirugía escritos en el siglo de oro cartagenero a orillas del mar Caribe, libros que sistematizaron la experiencia del ejercicio de la medicina en un puerto central de la Carrera de Indias y que fueron escritos ante todo por hombres innovadores e inquietos. Señalamos de todas formas, al igual que otros autores, la diversidad de ritmos históricos y niveles de desarrollo que plantean para su estudio los siglos XVI y XVII en el imperio de los Austrias. Las áreas científicas entonces existentes pueden dividirse en dos grandes grupos: el primero, integrado por saberes teóricos como las matemáticas, la cosmografía y la astrología, cristalizadas como discursos científicos a través de una larga tradición histórica; y el segundo, que tuvo un carácter muy heterogéneo, derivado de quehaceres pragmáticos que oscilaban entre el empirismo apenas organizado y la técnica sólidamente constituida, de origen autónomo, desarrollado en el seno social que cultivaba la tarea correspondiente. (López, 1979)

Como sostiene el historiador López Piñero, la medicina en este período ocupaba desde luego un lugar especial. De un lado, disponía de la tradición histórica más sólida, y de otro, constituyó un área que alcanzó autonomía social por su enorme importancia en el conjunto de la actividad científica de la época. Este campo del conocimiento, sin entrar en lo complejo de su estructura, tuvo múltiples relaciones con áreas teóricas como la historia, la filosofía natural y la cosmografía e incluso con la geografía y las matemáticas. (López Piñero, 2007)

El control eclesiástico de las producciones intelectuales no solo alejó a España y sus dominios del punto de partida de la revolución científica europea, sino que detuvo el avance experimentado durante el siglo XVI y la rica fase post renacentista en los países mediterráneos Lain Entralgo, 1972). Esto implica que sus saberes médicos y quirúrgicos se caracterizaban por dos grandes tendencias paradigmáticas: el escolasticismo arabizado bajomedieval y un emergente humanismo científico renacentista, opciones metodológicas que rivalizaban en la concepción del organismo humano saludable y los saberes acerca de la enfermedad y la intervención manual sobre el cuerpo. Estas dos tendencias, ligadas al papel progresivo de los árabes en el mediterráneo, contribuyeron a difundir los avances y hallazgos de médicos cirujanos italianos y franceses, entre ellos los del cirujano militar Ambrose Paré inspirador del cirujano Pedro López de León.

Del trabajo de López Piñero se desprende que, si bien gradualmente la cirugía buscaba una aproximación teórica a la medicina, en general, no se pudo evitar el colapso de la ciencia, y la medicina no fue una excepción (López Piñero, 1991). Quizá por eso, hasta finales del siglo XVII se llevaron a cabo reimpresiones de los textos escritos a finales del siglo XVI y comienzos del XVII, entre los que encontramos los tratados de los cirujanos romancistas como López de León, marcados por un galenismo moderado, que a pesar de conocer los avances del inglés Harvey frente 
a la circulación de la sangre, conocidos en 1616 y que significaban una ruptura frente al pasado medieval, se comportaban ante estos trascendentales avances con la precaución propia de las limitaciones impuestas por las jerarquías eclesiásticas y la Santa Inquisición². Tomando las palabras de Paula Ronderos en el siglo XVI y XVII, en España, se puede hablar de un renacimiento médico que incluyó dentro de la tradición galénica las obras de autores de origen islámico como Avicena y Mesue. Si bien es costumbre creer que la España de la Conquista, recién salida de la Reconquista, profesaba una aversión por todo aquello que fuese moro o judío, en el ámbito de la medicina, la cirugía y la farmacia se acoplaron las teorías útiles de ambas tradiciones culturales. (Ronderos, 2007, p. 175-196)

Centrándonos en lo anterior, nuestro trabajo pretende modestamente proponer la idea de que la llustración no fue el único momento de producción del conocimiento científico en el Nuevo Reino de Granada. Durante los siglos XVI y XVII, fundamentalmente en los puertos y ciudades conectadas al sistema de la economía mundo, se produjo un vital desarrollo del conocimiento científico y técnico. Los centros neurálgicos del poder político y comercial dan cuenta de ello durante el último tercio del siglo XVI y el interesante siglo XVII, sobre todo en lo que respecta a Lima, México, Veracruz y Cartagena de Indias, donde siempre estuvo presente la imperiosa necesidad de la realidad y se vivía un vigoroso combate entre la tradición y la innovación ${ }^{3}$.

A finales del siglo XVI, muchas fueron las dificultades para los avances de pensamiento científico en el Nuevo Reino de Granada. La sociedad y la cultura se vieron condicionadas por las políticas emanadas de la Contrarreforma. El rey Felipe II desplegó en Europa, África, y Asia un represivo proceso de evangelización que propició en la mayoría de sus extensos reinos, un estancamiento social. (Kamen, 1997). Procedimientos inquisitoriales de libros, como la indización de las obras científicas universales y el aislamiento ordenado por la Corona, frenaron el intenso combate entre tradición e innovación que nutrió los avances de la medicina, la cirugía, el arte de navegar, la ingeniería civil y militar y las matemáticas, en los siglos previos a la revolución industrial. (Rueda, 2014)

\section{LA ISLA DEL NUEVO MUNDO}

Como argumenta el historiador de la medicina Sotomayor Tribín la isla ecológica de la América prehis- pánica recibió el impacto de las invasiones trasatlánticas. (Sotomayor, 1997). En este experimento biológico a escala planetaria, los hombres europeos y africanos trajeron epidemias como la peste y la viruela, que diezmaron a las poblaciones originarias. A su vez, los blancos sufrían del vómito negro y todos en su conjunto estaban amenazados por la lepra y la sífilis, sin olvidar la conformación de una sociedad esclavista con condiciones laborales extremas y donde los accidentes abundaban, poniéndose en riesgo continuo la inversión en la mercancía humana.

La Carrera de Indias fue un experimento que formalizó las relaciones comerciales trasatlánticas entrelazando entre si varios continentes, lo cual facilitó que las epidemias se expandieran con rapidez. Las flotas de galeones, las naos de esclavos y otras mercancías facilitaron su aparición. La malnutrición, la falta de higiene y las picaduras de insectos hicieron el resto golpeando a la población portuaria y, sobre todo, a las tripulaciones. Desde 1541, cuando se estableció el monopolio comercial, Cartagena de Indias quedó como puerto único de escala hacia el virreinato del Perú y desde entonces empezó a recibir flotas de armadas y galeones periódicamente, volviéndose así propensa a este riesgo.

Escasos historiadores conceden a Cartagena de Indias importancia desde el punto de vista científico y cultural en el siglo XVI y XVII, como si se entendiera que todas las condiciones sociales dadas parecían vedadas a las prácticas de los saberes modernos. Sin embargo, no tienen en cuenta que desde finales siglo $\mathrm{XVI}$ y hasta la primera mitad del siglo XVII, ejerció como el puerto más importante del imperio en el Caribe y, por ende, como un centro de gestión comercial trasatlántica, avanzada financieramente (Bernal, 1992), celosamente protegida y con poderosas casas comerciales instaladas (Vidal, 2002). Todo ello brindó las condiciones necesarias para el desarrollo de una estructura hospitalaria y un excelente marco de actuación de reconocidos médicos y cirujanos. (Solano , 1998)

Entendemos válido y pertinente hacer el esfuerzo en esa dirección. A finales del siglo XVI, en la ciudad puerto se escribieron dos tratados de medicina. El primero, más conocido y objeto de atención incluso por el nobel Gabriel García Márquez, Los discursos medicinales de Juan Méndez Nieto, escrito en Cartagena de Indias en 1607 con fines didácticos y educativos de las prácticas médicas y con una valiosa información etnográfica que además ofrece un fresco 
variado de la vida cotidiana de la ciudad. (Hernández, 2011, p. 93-117)

El segundo, en el que centramos nuestra atención, escrito por el cirujano de la ciudad de Cartagena de Indias Pedro López de León, quien después de una dilatada trayectoria profesional en los abigarrados puertos de Sevilla y Cartagena a ambos lados del océano, escribió Práctica Teórica de los Apostemas, en general y particular cuestiones prácticas de cirugía publicado en Sevilla en 1628 (Solano, 2007). Por este tratado fue referencia de la cirugía durante el siglo XVII y su obra, reconocida y difundida en el imperio hasta inicios del siglo XVIII, ya que practicó una de las líneas más progresivas de la cirugía renacentista española y europea, la Vía particular. (López, 2007, p. 408)

La propuesta de López, que seguía a su maestro Agüero, apostaba por la técnica de la vía particular o secante, innovación que acogieron ambos cirujanos sevillanos en el Hospital del Cardenal de Sevilla. Esta opción tuvo dos fuertes contradictores: el cirujano de la Universidad de Alcalá Juan de Fragoso y Dionisio Daza Chacón cirujano de Felipe II. Ambos se desmarcaban de la propuesta de desecar las heridas y cerrarlas mediante puntos y apostaban por la vía común que privilegiaba el Pus Loable L $^{\text {. }}$

El conocimiento de la existencia de la obra y la escasa atención prestada a su autor, sobre todo en Colombia, nos motivó a revisarla para entender su significado y comprobar que se trató de un intento de construir conocimiento científico con base en un escenario de urgencia y necesidad de vida como lo fueron los puertos, donde la vida era el comercio y este jamás debía detenerse.

\section{EL EJE PORTUARIO CARTAGENA DE INDIAS- PORTOBELLO Y SUS GRANDES FERIAS COMERCIALES}

A mitad del siglo XVI, los corsarios europeos atacaban las rutas comerciales y los puertos españoles en toda la cuenca del Caribe, acciones mantenidas gracias a una red de refugios y puntos de abastecimiento en pequeñas islas, y, sobre todo, en las inabarcables costas continentales. La Carrera de Indias atrajo a los enemigos del imperio y con ello el peligro al creciente número de barcos provenientes de España. Ello obligó a instaurar un sistema convoyar de flotas. (Rodríguez, 2015)
La consolidación de una economía minero-extractiva en la América española provocó un aumento de la producción de metales preciosos -plata y en menor medida oro- y ello tuvo efecto en el comercio trasatlántico y el número de barcos que componían las flotas. En 1564, las flotas se dividieron en dos: una, la de Nueva España, con destino las ferias de Veracruz y otra, hacía Cartagena de Indias y las ferias del Istmo, llamada la flota de Tierra Firme.

Durante la segunda mitad del XVI hubo un incremento en la actividad portuaria que sustituyó a Santo Domingo como lugar central del comercio con España por el complejo portuario Cartagena-Portobelo, convertido en el eje articulador del espacio regional del Caribe como lo demuestran las cifras del comercio. (Chaunu, 1997. García de León, 2017)

La fragilidad de los fondeaderos del Istmo y la precariedad de condiciones para albergar un puerto funcional benefició a Cartagena de Indias. El desarrollo de este complejo portuario hubiera sido impensable sin esta; fue guardiana y defensora de la ruta de la plata y sus ferias. En realidad, como expresó Chaunu, fue un puerto levantado para la escala, un puerto de guerra.

Como puerto central de las rutas oceánicas ejerció funciones de control fiscal, administrativo y militar, con el consiguiente crecimiento de un mercado local que le proporcionó liderazgo económico a comienzos del siglo XVII. La función geoestratégica y comercial generó un ambiente social que acogió una abundante población extranjera, llegando este colectivo a participar de manera determinante en todos los ámbitos de la sociedad y de manera especial en su contratación. (Vila, 1979, p. 147-184)

El Obispo Juan de Ladrada lo expresaba en una carta del 22 de junio de 1599:

El haber hecho esta ciudad tan grande y populosa, y haberse avecindado en ella tanto forastero, ha sido lo principal las galeras, porque se gastan muchos mantenimientos y con lo que se gasta y consume en ella de situado cada año, se hacen ricos muchos hombres; y esto no disminuye la hacienda real si no que la acrecienta, pues crecen los derechos reales creciendo el comercio. ( Martínez, 1986, p. 174)

Gracias al desarrollo del comercio a larga distancia, como puerto de armadas y flota primero y luego como puerto único negrero entre 1580 y 1640, se transformó en una ciudad de servicios. Los gobernadores responsables de la ciudad tenían contraída la 
obligación de defender y abastecer el puerto en lo relativo al buen funcionamiento del comercio como centro de reabastecimiento del servicio de comunicaciones con la metrópoli. Fray Antonio de Hervía en 1589 decía "Que proveer esta tierra a tan grandes flotas, galeras, soldados, y mantenerse así, no es pequeño negocio" (Martínez, 1986, p. 166). La diversidad de las prácticas comerciales que se desarrollaron en este tiempo y sus condiciones naturales, la hicieron una pieza básica en la estructura del comercio Atlántico. La trata, el cacao, el añil, el tabaco, las perlas, los cueros y maderas, entre otros, encontraron un lugar privilegiado de lanzamiento para salir al mercado tanto europeo como americano. En este contexto llegó el Cirujano de Indias, para ocupar un destacado lugar como médico clave para la supervivencia de una ciudad-puerto expuesta a continuas epidemias y enfermedades.

\section{PEDRO LÓPEZ DE LEÓN, EL CIRUJANO DE LA CIUDAD DE CARTAGENA DE INDIAS}

El cirujano Pedro López de León fue partícipe activo de los debates que se adelantaron en su tiempo frente a la Medicina y la Cirugía, y que conciernen en la vía particular, y vía Común. No tenemos mucha información de los primeros años de su vida, aunque sabemos que nació en Sevilla a mediados del siglo XVI y que, siendo estudiante de la Universidad, practicó la cirugía con el médico Bartolomé Hidalgo de Agüero de quien aprendió el sentido clínico, la destreza quirúrgica y el método de la vía seca. Concluida su carrera, y tras unas breves prácticas, ocupó la plaza de primer cirujano en el Hospital del Cardenal de Sevilla desde 1578 hasta 1590, año en el que fue nombrado cirujano real de galeras y fue enviado en la flota de Tierra firme con destino a Cartagena de Indias. (Hernández, 1960, p. 5)

En este puerto caribeño vivió los mejores años de su vida profesional y dio continuidad a los presupuestos de su maestro, enriqueciendo su legado con la experticia adquirida por casi 30 años, la mitad de su vida, mientras ejercía la medicina en un puerto central de la Carrera de Indias, tiempo en el que escribió Práctica y Teórica de apostemas en general, y particular. Por el licenciado Pedro López de León, cirujano en la ciudad de Cartagena de Indias. En la cirugía, su posición era la de usar la vía particular alternativa basada en la opción secante y aséptica para la cura de heridas frescas, que rompía con las tendencias tradicionales basadas en el uso de emolientes.

Como afirma Hugo Sotomayor, la obra de López de León, al igual que la de su mordaz antecesor y también contemporáneo en Cartagena, el médico Méndez Nieto, contrasta con la ausencia de una similar escrita en cualquier otro lugar de la Nueva Granada durante los dos primeros siglos de la colonia (Sotomayor, 2009). Lo único parecido con lo que se puede comparar es con la que escribió en Santafé de Bogotá el jesuita Pedro de Mercado, que publicó en Sevilla en 1680 Recetas de espíritu para enfermos del cuer$p o^{6}$. Si bien las primeras permiten al investigador ver la claridad y brillantez alcanzada por la medicina española en el siglo XVI, la segunda deja entrever el poder que tuvo el aparato religioso durante el siglo XVII sobre el ejercicio médico profesional y su terrible influencia sobre los conceptos de enfermedad y salud. Cartagena de Indias, por su conexión al mar durante el periodo colonial, siempre se anticipó al conocimiento de los avances científicos y de las nuevas ideas políticas. (Sotomayor, 2003, p.125-143.)

Pedro López de León fue heredero no solo del legado teórico de Hidalgo, sino de un dispositivo técnico que le brindó la pericia manual y experimentación de sus teorías, practicadas frente a un mosaico de razas y enfermedades en un puerto caribeño sin imaginarnos la sociedad que dejó atrás en la Península ibérica. Ello deja entrever la trascendencia de una obra que lo elevó a figura principal de la cirugía durante el claro oscuro del barroco.

Publicó su obra en medio de una época difícil para el estímulo científico, pues la Corona, en su afán por aplicar la Contrarreforma en todos los rincones del imperio, llevó hasta el extremo el papel mesiánico de la Evangelización del nuevo Mundo que cedió a la Inquisición. Dicha obra representa para el siglo XVII español la prolongación del legado de la cirugía renacentista de vanguardia (García, 1976). Además de su empirismo, en su trabajo se condensa no sólo la influencia de los clásicos griegos redescubiertos por el humanismo, sino los aportes teóricos y técnicos alcanzados en Europa.

Vivió y ejerció la profesión en Cartagena de Indias, aunque el ejercicio de su destreza lo llevó a otros puertos, como al de Santa Marta. Fue uno de los autores más leídos en la península en el siglo XVII y su prestigio atravesó fronteras, haciéndose conocido en distintas regiones de Europa, y sus tesis perdura- 
ron hasta el cambio de paradigma de la cirugía producido en el siglo XVIII. Por esta razón, los historiadores de la medicina López Piñero y García Ballester lo consideran el más importante cirujano español del XVII, puesto que prolongó el legado de Hidalgo de Agüero y en medio de las sombrías y confusas circunstancias, representó el límite superior de la cirugía española de entonces.

Cartagena de Indias, equiparada entonces a los avances europeos, fue un centro que brindaba los avances de la cirugía moderna en Tierra Firme y el Nuevo Reino gracias a López de León. Por otra parte, las circunstancias propias de la ciudad, volcada hacia el exterior y con una población flotante de gentes de todas las razas y confines, hacían propicia la coyuntura para ser un verdadero centro de experimentación y confrontación de sus teorías, puesto que el primado de la práctica caracterizó la labor de López en el Hospital de Cartagena.

Lo explica en su libro de la siguiente forma:

“En el hospital de Cartagena de las Indias se curan de bubas cada año, quinientos enfermos o poco más o menos y a que curo en él 23 años...en las ocasiones que se ofrecen de Flota y Armadas que a éste puerto vienen... suele haber ciento cincuenta y doscientos enfermos, y como aquí acuden tantos baxeles de Guinea y otras partes, siempre está el hospital lleno : demás que de Panamá y Puerto Belo vienen a este hospital y assi mismo de Santa Fe y de todo el Nuevo Reyno, y assi mismo vienen de Santa Marta, Río de la Hacha, Caracas, Margarita y de todas las Islas de Barlovento a fama de las grandes y estupendas curas que en este Hospital se hacen de todas las enfermedades." (López de León, 1628)

\section{UNA APROXIMACIÓN BIBLIOMÉTRICA A LA PRÁCTICA Y TEÓRICA DE LAS APOSTEMAS EN GENERAL Y PARTICULAR ${ }^{7}$}

Pedro López de León vivió en un tiempo de control, no sólo de la lectura de libros extranjeros, sino principalmente de autores españoles que debían pasar la censura eclesiástica. Esta expurgaba los contenidos de acuerdo a la doctrina católica: el doctor Román, quien tuvo a su cuidado la lectura del tratado de López de León por comisión del Vicario de la Villa de Madrid, comentaba así el contenido del libro en la introducción:

Aviendo se me cometido por el señor Vicario de la villa de Madrid, el examen de la obra del licenciado Pedro López de León, Medico y ciruxano vezino de la ciudad de Cartaxena de las Indias, que trata de la Teórica y Práctica de las apostemas en general y en particular de las Questiones prácticas de cirugía y de las heridas y llagas y aviéndole visto con particular diligencia: Hallo ser la doctrina no solamente sana y muy catholica a más de la grave y provechosa que se aya escrito en mi lengua española, y que muestra el autor aver trabajado y visto muchos autores griegos y modernos que tratan de cirugía y porque esto me parece, lo firmo en mi nombre ${ }^{8}$.

La obra usa un método lógico formal que, aunque fundamentado en principio en los criterios de autoridad de Aristóteles, no permanece sólo en el ámbito deductivo heredero de la escolástica, sino que promueve la inducción como elemento que sustenta verdades generales. Propone una dialéctica TeoríaPráctica-Teoría que se asemeja al clásico método científico positivista. El libro, conforme a su tiempo, está concebido con una parte general y una particular que se traduce en una síntesis de reglas generales al final.

A continuación, usaremos las citas bibliográficas que aparecen en la obra para acercarnos a las lecturas que utilizó para elaborar el libro y con ello ver la información médica que usó durante el ejercicio de su profesión.

Tras analizar los datos podemos sostener que su contenido prolonga los hallazgos de la medicina renovadora del Renacimiento, aunque es evidente el sesgo del legado greco árabe: En síntesis, la obra es resultado de la larga tradición mediterránea en los inicios de la primera modernidad de la ciencia. Estudiando las citas bibliográficas del libro como referente principal aparece Galeno (27.02\%), a continuación, se destaca Avicena (10.4\%) pero no se puede soslayar el uso que hace del Corpus Hipocrático (10.35\%). Sobresale la presencia innovadora del discurso quirúrgico español e italiano de la época: de la Península Ibérica cita cirujanos de la renovación como Fragoso, Dionisio Daza, Alcázar, Falcón y Juan Calvo (10.12 \%) sin olvidar a Bartolomé Hidalgo de Agüero. Usa referentes de la Universidad de Alcalá, como Vega y Luis de Lemos. Italianos y franceses como Ambrosio Paré, Bartolomé Maggio, Giovanni Da Vigo, Dino de Florencia, Antonio Musa Brassavola, Gianbattista Theodosio, Valleriolla, Nicolao Massa, Andrés Mattiolli y Gianbattista Montagnana, que suman el $6.85 \%$ de las referencias. Otros autores árabes mencionados son Rhazes, Avenzoar, Alfarabio, Mesué y Ali Abbas, entre los que destaca el cirujano hispano Abulcasis. (5.68\%). 
Tabla tomada del libro Salud, cultura y sociedad en Cartagena de Indias siglos XVI y XVII.

Tabla de autores citados por López De León

\begin{tabular}{|c|c|c|c|}
\hline Autor & $\%$ & de cita & Textos \\
\hline Galeno & 27,02 & 347 & $\begin{array}{l}\text { Methodum, De usu partium, Artis curativa ad glauconem, De ventri, } \\
\text { Tegni, De morbis curandis de curam ratio per sanguinis missio, De } \\
\text { oculis, Inaequali intemperies, De differentis febri, de diferentis morbis, } \\
\text { Prognosticorum, De prefagione ex pulsibus, De locis affectis, Artis } \\
\text { medicinae, De sanitate, De venum affecti, De Compositione, De locis } \\
\text { affectis, Medicamentorum secundum locos, De compositione } \\
\text { medicamentorum secundum genera, De fracturis, De compositione, } \\
\text { De simplic. med. facult., De membrorum, De natura humana, De } \\
\text { compositione pharmacorum, Comentarium, Pronosticum, Aphorismi. }\end{array}$ \\
\hline Avicena & 10,4 & 134 & Canon, Sentencias, Tratado \\
\hline Hipócrates & 10,35 & 133 & $\begin{array}{l}\text { Corpus hippocraticum: De fracturis, De ulceribus, De morbis, } \\
\text { Epidemiorum, Aphorismi, De vulneribus capitis, De internis } \\
\text { affectionibus }\end{array}$ \\
\hline $\begin{array}{l}\text { Modernos españoles: Laguna } \\
\text { Alcázar, Falco, Fragoso, Juan } \\
\text { Clavo, Dionisio Daza, Bartolomé } \\
\text { Hidalgo de Agûero }\end{array}$ & 10,12 & 130 & $\begin{array}{l}\text { Alcázar: Andreae Alcazaris medici guadalakarensis in amplissima } \\
\text { Salmanticensi Academiae Chirurgiae facultatis Libri sen (MDLXXV). } \\
\text { Falco: Práctica de cirugía de Juan de Vigo. Traducción (1537). } \\
\text { J. Fagroso: Cirugía Universal (1581). J. Calvo: Cirugía (1580). D. Daza: } \\
\text { Prácticay teória de cirugía. B. Hidalgo de A: Thesoro de la verdadera } \\
\text { cirugía (1606). }\end{array}$ \\
\hline $\begin{array}{l}\text { Paulo de Eginay Aecio, Dribasio y } \\
\text { Alejandro de Tralles }\end{array}$ & 9,19 & 118 & Memorándum (Hypomnema). Aecio: Tetrabiblon \\
\hline $\begin{array}{l}\text { Modernos italianos y franceses: } \\
\text { Bartolomeo Maggi, Ambrosio } \\
\text { Paré, Giovanni da Vigo, Nicolao } \\
\text { Massa, Dino de Florencia }\end{array}$ & 6,85 & 88 & $\begin{array}{l}\text { B. Maggi: De vulnerum a bombardarum et scopetorum globulis } \\
\text { illatorum et de eorum syptomatum curatione tractatus (1552). Ambrosio } \\
\text { Paré: La methode de traicter les playes faictes par hacquebutes et } \\
\text { oultres bastons à feu: et de celles qui sont faictes parb flèches, dards } \\
\text { et semblables (1545). G. Da Vigo: Practica in arte chirurgica copiosa } \\
\text { continens novem libros (1557) }\end{array}$ \\
\hline $\begin{array}{l}\text { Guy de Chauliac, Guillermo de } \\
\text { Saliceto, Hugo de Lucea, } \\
\text { Theodorico de Lucoa, } \\
\text { Lanfranchi, Arnau de Vilanova }\end{array}$ & 6,69 & 86 & $\begin{array}{l}\text { G. Chauliac: Chirugia Magna (1363), al menos } 7 \text { ediciones, en catalán } \\
\text { y, romance entre } 1463 \text { y 1597. G. de Saliceto: Cyrurgia, Summa } \\
\text { conservationis et curationis. Hugo de Lucca: Esc. de Bolonia. } \\
\text { Lanfranchi: La cirugía del maestre Lanfranchi mediolanense, Sevilla, } \\
1495\end{array}$ \\
\hline $\begin{array}{l}\text { Rhazes, Abulcasis, Alfarabio, Ali } \\
\text { Abbas, Mesué Avenzoary } \\
\text { Averroes, Azarabio }\end{array}$ & 5,68 & 73 & $\begin{array}{l}\text { Katib Altasrif: Concesio ei data qui componere haud valet. Ali Abbas: } \\
\text { Tractatus de medicina (Liber sanitatis, Liber morbi, Liber signorum, } \\
\text { Liber regius). Averroes: Liber universalis de Medicina. Mesué: } \\
\text { Antidotarium. }\end{array}$ \\
\hline Celso, Dioscórides, & 5,29 & 68 & $\begin{array}{l}\text { Celso: De re medica (sobre la medicina). Discórides: Hylika; De materia } \\
\text { medica. }\end{array}$ \\
\hline $\begin{array}{l}\text { Vesalio, Juan Valverde, Silvio } \\
\text { Jacobus, Realdo Colombo }\end{array}$ & 2,95 & 32 & $\begin{array}{l}\text { Vesalio: Fabrica, Valverde: Historia de la composición del cuerpo } \\
\text { humano. Colombo: De re anatomica }\end{array}$ \\
\hline Clásicos de Greciay Alejandría: & 1,55 & 20 & Escuela de Alejandría \\
\hline Filósofos: Aristóteles yPlatón & 1,40 & 18 & Phisica (Libro Vll), De animalibus, De anima, Metaphisica. \\
\hline $\begin{array}{l}\text { Pietro } \text { C’ Abano, Rogerio, Gilles } \\
\text { de Corbeil, Valesco de Taranta }\end{array}$ & 1,24 & 16 & $\begin{array}{l}\text { P. D’Abano: Conciliarum controversiarum quae inter philosophos et } \\
\text { medicos Versantur. Rogerio: Practica chirurgiae. Gilles de Corbeil: } \\
\text { Liber de laudibus et virtutibus compositorum. Valesco de Taranta: } \\
\text { Medicaminum Philonium pharmaceuticum et chirurgicum medendis } \\
\text { omnibus cum internis tun externis humani corporis affectionibus (1418). }\end{array}$ \\
\hline \multirow[t]{2}{*}{$\begin{array}{l}\text { Terapias medicamentosasy } \\
\text { antidotarios: Michelangelo } \\
\text { Blondo, Antonio Calmetheo, } \\
\text { Andrea Mattioli, Antonio Musa } \\
\text { Brassavola, Juan Andrés de la } \\
\text { Cruz, Luis de Lemos }\end{array}$} & 1,16 & 15 & \\
\hline & 99,8 & 726 & \\
\hline
\end{tabular}


Cita clásicos latinos y griegos. Encontramos dentro de los primeros, a Dioscórides y Cornelio Celso (5.29 $\%)$, entre los bizantino-alejandrinos aparecen Aecio, Oribasio y Alejandro de Tralles y en especial Pablo de Egina (9.19\%), cuyos textos quirúrgicos todavía tenían audiencia y reputación. Presenta autores redescubiertos en el Renacimiento, tales como Herófilo, Erasístrato y Eudemo (1.55\%), que acompañan a Aristóteles y a Platón, quienes brindan el marco de la lógica y la reflexión filosófica (1.4\%).

Los clásicos tienen una presencia cercana a la de los de su tiempo y lo inscriben, a pesar de su acento técnico mediterráneo dentro del humanismo revivido del siglo XVI. Las relaciones de causalidad establecidas, así como la indispensable deducción e inducción, lo general y lo particular, el género propio y la diferencia específica, fueron operaciones extraídas de la filosofía aristotélica.

Entre los soportes más fuertes en lo concerniente a la cirugía, están las escuelas de Paris y Montpellier presentes con Guy de Chauliac, tras del cual encontramos la sombra de Guillermo de Saliceto, Hugo y Teodorico de Lucca y la tradición de la cirugía medieval vinculada a Bolonia, a la cual se pueden sumar Lanfranchi y en alguna medida, Arnau de Vilanova representados en el $6.69 \%$ de las citas, que sumados al 1,24\% que representan Valesco de Taranta, Pietro D’Abano, Gentile da Foligno, Rogerio y Gilles de Corbeil, denotan una línea relevante.

Muestra en su obra una absoluta claridad acerca de los caminos que transitaban la anatomía vesaliana y posvesaliana. Aunque se deja seducir a veces por la tendencia de la época, donde predominaba el autor de La Fábrica de Vesalio, no desconoce la gran obra crítica del español Valverde de Amusco, puesto que los cita en conjunto con Renaldo Colombo y Silvio Jacobus, también rivales de Vesalio e inspiradores del primero. En todo caso, usa con asiduidad a estos anatomistas a los que suma, en un sentido más general, a los francesesTagault y Houllier que suman en total el $2.95 \%$ de las citaciones bibliográficas.

Otros autores, como Antonio Calmetheo, Michelangelo Blondo, el propio Laguna y Mattioli influyen en lo médico y lo farmacéutico. También encontramos a Antonio Musa Brassavola, Juan Andrés de la Cruz y Luis Lemos que representan un porcentaje que llega al $1.16 \%$. Por último, se referencian autores de otras épocas como Sorano, Mariano Santo, Leonitello, Bruno... etc.

En resumen, a lo largo del tratado se referencian casi medio centenar de autores de primera línea, lo cual evidencia que Pedro López de León era un profesional actualizado de su tiempo. Con seguridad, su biblioteca médica debió estar en Cartagena, como sabemos de su propia versión que también estaba la de Juan Méndez Nieto. ${ }^{9}$

Desde el punto de vista de la literatura humanista, se aprecia un manejo probable de las traducciones de Francisco Valles (1577), o incluso los trabajos de Pedro Jaime Esteve (1551) sobre el texto de las Epidemias de Hipócrates, o los trabajos de Laguna sobre Dioscórides.

Apoyado en su nutrida biblioteca, López de León reclamaba un espacio inédito para el ejercicio de la cirugía y así desalojar a barberos y albéitares, que actuaban empíricamente sin formación académica. En este sentido, los médicos y cirujanos como Pedro López de León empezaron a transitar un nuevo camino en una ruptura categórica con el pasado. No obstante, hay que resaltar su propósito de ampliar el auditorio a los llamados cirujanos romancistas, personal autodidacta que no tenía oportunidad de asistir a la academia ni poseía conocimientos de latín o griego pero que requería complementar su formación. Para ellos también escribían los científicos de entonces, en lengua romance, que fue la búsqueda de un nuevo soporte para la implantación de este nuevo discurso, que luchaba entre tradición y renovación.

La lectura, en lo relativo a lo técnico, permite detectar aspectos específicos vinculados con lo que él denomina obra de manos, que nos deja ver su habilidad y experticia en el tratamiento del cuerpo humano. Esta pericia, exhibida con seguridad y autoestima, queda plasmada en el libro con un sentido claramente pedagógico.

Como soporte de este discurrir técnico encontramos toda una base conceptual clásica, reformulada por los modernos a la cual añade su experiencia. El propio autor deja claros sus propósitos generales en el texto:

- Sustentar la argumentación y la acción en criterios de autoridad teórica, esencialmente médica más que quirúrgica, en virtud de lo cual se acude a los clásicos rescatados por la renovación humanista para legitimar y actualizar el discurso.

- Proporcionar definiciones positivas con un grado de elaboración científica que sitúe a la cirugía en un nuevo terreno, superando el empirismo que caracterizó su fase pre-sistemática.

- Apoyar en elementos de la lógica formal el orden del discurso y la exposición pedagógica a su población objeto: los cirujanos romancistas. 
Influencias teóricas de Pedro López de León

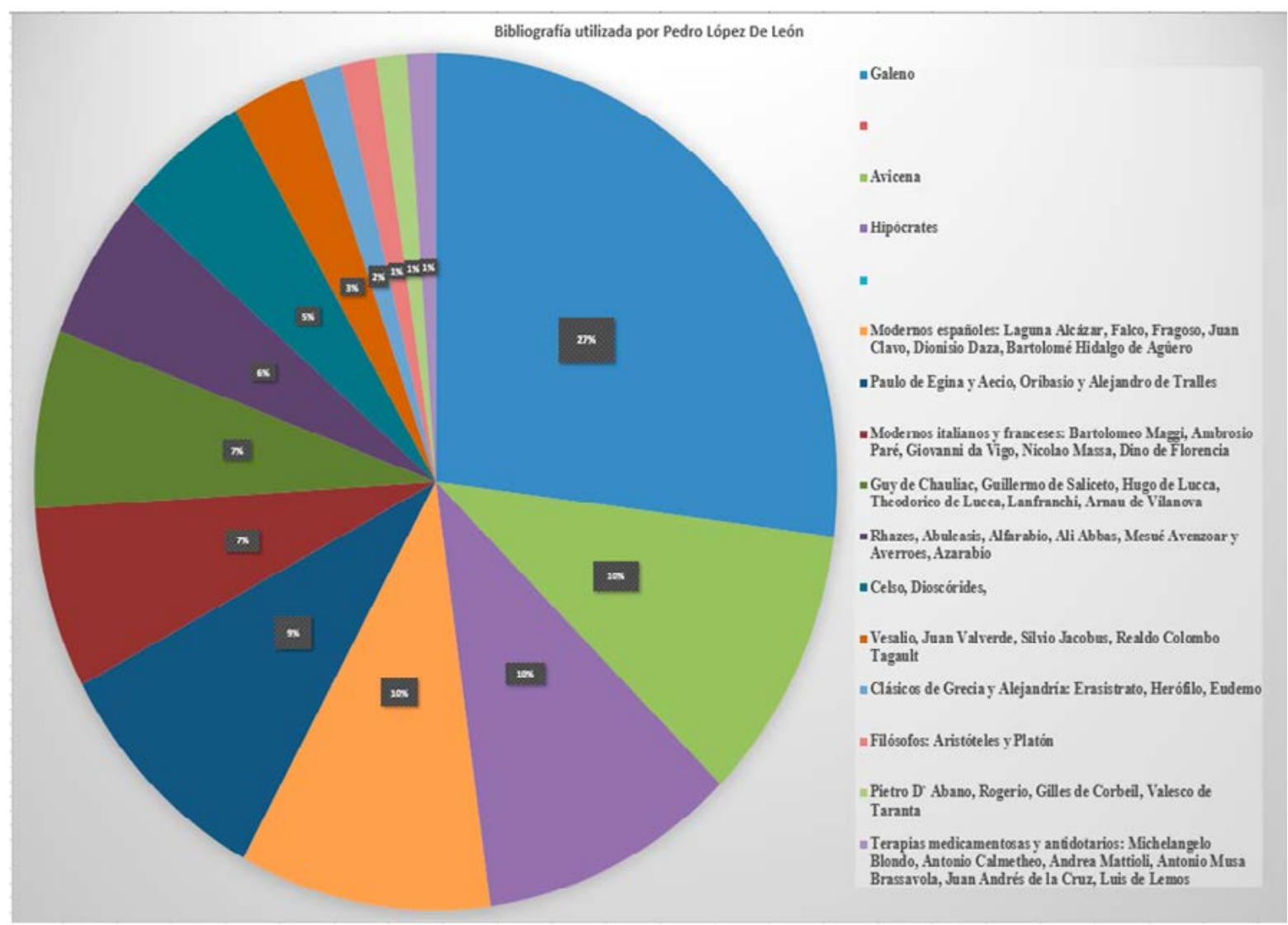

Fuente: Elaboración propia

- Propiciar el debate con los cirujanos y médicos practicantes y con los entendidos en la teoría.

Son fácilmente distinguibles en el libro cuatro partes distintas y complementarias: Fase teórica, contenida en los tres primeros Libros. Fase Polémica, en la Segunda Parte "El Agregado de Cirugía". ase técnica, que discurre de los Libros III, "De las Llagas Frescas,"; IV, "De las heridas del vientre y región del Abdomen"; V, "De las Ulceras"; VI, "De las Fracturas y Dislocaciones" y libro VII, “ Del morbo Gálico". I antidotario

\section{REFLEXIONES DE LA PRÁCTICA DE PEDRO LÓPEZ DE LEÓN Y SUS INSTRUMENTOS QUIRÚRGICOS Y DE LABORATORIO}

La referencia a la praxis se sustenta en el grado de importancia que concedía como factor de confrontación a la teoría. Sus ideas quedan claramente expues- tas en el apartado que denomina "Reglas de Cirugía" y donde plasma tres propósitos científicos elocuentes:

- "La práctica es una obra que se acomoda con las reglas y leyes de la teórica".

- "La salud no se restituye con palabras sino con remedios tomados como conviene."

- "La ciencia sin la experiencia no acarrea mucha confianza de médico para paciente". ${ }^{10}$

Con referencia al oficio de médico, entendido como profesión moderna y distante de las posturas taumatúrgicas sostiene, "El oficio del buen médico, es sanar la enfermedad, o por lo menos reducirla a mejor estado o en aquel que la Naturaleza es capaz." (López, 1692, p. 368) Los médicos que buscaban la aceptación y aprobación de las autoridades reales también se transparentan en este juicio: "El artífice que desea hacer alguna cosa grande y digna de alabanza, ha de 
obrar con mucha diligencia en el conocimiento del sujeto propio". (López, 1692, p. 368)

Esta postura socrática no excluye la pedagogía del arte médico "El que no asistió muy de ordinario a las obras del arte y a las lecciones, de los Doctores, y que sólo por aver leydo mucho se vende por Cirujano noble, mucho se engaña y es desvergonçado." (López, 1692, p. 368)

Es clara la complementariedad de la teoría y la práctica que exige López de León: "El que huviere acarreado para si el magisterio de la cirugía con dineros y no con el uso, jamás hará cosas que sean dignas de alabanza," (López, 1692, p. 368) en síntesis el imperativo es: "Conviene que el cirujano sea ágil y que tenga industria y sea de muy buenas manos y que no se fíe en los libros." (López, 1692, p. 368)

Queda evidenciado su actualización en la discusión médica de la época, en la cual prevalecía la lógica de la escolástica, o bien el pragmatismo de los empíricos. Por el contrario, se abría paso la academia como una reconceptualización radical de la cirugía, línea que representaba el sevillano. Deja clara su preocupación por la necesidad obligada de erradicar a los impostores, revela así mismo que los cirujanos empíricos y barberos empezaban a ser desplazados por médicos cirujanos, de los cuales él es una muestra.

Recomienda que los prácticos deben reconocer una necesaria jerarquización:

“...deviendo los cirujanos, boticarios y barberos reconocer a los señores médicos por superiores en la medicina y la cirugía y en elección de drogas y todo lo demás, que en cuanto a la teórica saben más en todas las facultades dichas que el cirujano ni el boticario si no es que el cirujano es médico y aunque lo sea siempre es inferior quanto al ejercer el arte, mayormente si el médico está graduado de doctor que tiene en la medicina mucho más autoridad como consta por sus títulos." (López, 1692, p. 20)

Es evidente la preocupación por la situación que atravesaba el ejercicio del arte médico y farmacéutico en las Indias hacia donde se desplazaban una gran cantidad de aventureros y charlatanes. Es cierto que la Institución del Protomedicato arbitró mecanismos de control y legislación al respecto. No obstante, cuestiona la calidad de los Protomédicos de Tierra Firme como comprobamos en varias ocasiones a lo largo de su obra. Denunciaba a las autoridades locales que permitían el abuso y el desorden de personas ajenas al conocimiento médico profesional, tales como albéitares, barberos, falsos boticarios, mohanes y comadres, personajes abundantes en las inmediaciones del puerto. Por ello mantiene la posición de exigir títulos de médicos universitarios, lo cual consideraba crucial para la vitalidad de la república

Su reflexión y sumada a ella, las irónicas críticas de Méndez Nieto, les sitúan en la perspectiva de profesionales que quieren dignificar la actividad médica venida a menos con los excesos y ligerezas del proceso conquistador, para con ello afianzar instituciones de salud confiables.

Lo que diferenciaba a Pedro López de los llamados "médicos latinos" consistía en que éstos últimos acudían esencialmente a la teoría, pero se mantenían distantes de la práctica, manteniendo la separación entre trabajo manual e intelectual, tradición procedente de la antigüedad grecolatina.

Por el contrario, López de León y la escuela sevillana reconocían el valor de la "obra de mano" y con ello los médicos cirujanos empezaron a desarrollar un instrumental obligado para intervenir el cuerpo humano, consolidándose con ello el reconocimiento de la práctica. La tradición de Pedro López reconoció tres tipos de instrumentos técnicos para el ejercicio de la cirugía, algunos procedían de la medicina castrense y acompañaban a cirujanos militares destinados a las galeras. Utilizaba tres tipos de instrumentos:

- Instrumentos ferrales para las intervenciones quirúrgicas.

- Instrumentos de laboratorio para la práctica de preparaciones químicas curativas.

- Instrumentos ortopédicos para las afecciones de los huesos: fracturas, dislocación, etc.

Este aspecto lo consideró tan importante, que la primera edición de su libro en 1628 anuncia la presentación gráfica del instrumental quirúrgico para sus lectores, especialmente para los cirujanos romancistas. Observemos la explicación que nos brinda:

“...he puesto dibuxados en las láminas al fin del libro a número 28 para los cirujanos romancistas, esta variedad de cauterios actuales, que los authores traen para diferentes partes de nuestro cuerpo, y unos sirven para las llagas podridas de las canillas y piernas, los quales son quadrados y triangulares. Otros hay como media caña, para las canillas y huesos de los bracos. "(López, 1692, p. 298)

Prosigue señalando otros tipos de cauterios que llama "datilares," para el miembro viril y otras úlceras; otros incisorios llamados cuchillares para cortar y otros para abrir los abscesos de diferentes formas $y$ 
tamaños; otros para abrir apostemas entre las costillas, en "la cavidad vital" y en el hígado, también para las hernias y de otros tipos. Diversos tipos de puntas, incluso algunas especiales para las mucosas y los ojos.

Sostiene con tono pedagógico, pero no sin firmeza, que el cirujano debe estar provisto de todos los instrumentos que puedan ser necesarios. Concluye estableciendo la diferencia con otros practicantes del arte: "y el cirujano que le faltan las herramientas que su arte manda, no le llaméis sino medio cirujano, que este nombre merece como el carpintero sin su hachuela". (López, 1692, p. 298) Finaliza el libro dibujando una tabla con todos los instrumentos y detallando el uso de cada uno.

Si bien el manejo de los problemas ligados a la ortopedia, dislocaciones y fracturas óseas las expone en el Libro Sexto-De las fracturas y Luxaciones, es preocupación de la época clásica. Esto queda plasmado en el libro en la clasificación de utensilios que dibuja, explica y anexa al final. Para cerrar el libro, centró su atención en los Hornos y elementos de lo que llamaba la latroquímica ${ }^{11}$, utensilios que reflejan el grado de avance y experimentación de su conocimiento, pues con ellos está presente el proceso de transformación de sustancias botánicas para la búsqueda de remedios a las enfermedades, donde experimentó con plantas europeas y americanas. Hornos, alambiques, baldes, entre otros, detallados minuciosamente en sus dibujos refuerzan la idea, una vez más, de la práctica y la teoría.

\section{A MANERA DE REFLEXIÓN FINAL}

Para cerrar el presente trabajo de análisis y lectura del libro de Pedro López de León, queremos señalar algunas reflexiones para sugerir pensar la historia del siglo XVII de una manera más compleja en relación al compromiso de los profesionales con sus saberes y con la producción de conocimiento científico que, sin señalar cambios dramáticos en la evolución del conocimiento, hicieron sus aportes en el punto de partida de la ciencia moderna occidental.
Con nuestro modesto trabajo, hemos querido sostener que, a veces, este siglo se ha estudiado sin la profundidad necesaria, o más bien, se han hecho generalizaciones simplistas en torno a él. Esto ha ocasionado la omisión de cualquier desarrollo científico en general en Iberoamérica, y en particular en Colombia, en el tiempo de la configuración de las sociedades criollas, con el peligroso sesgo ideológico que excluye a nuestra historia del punto de partida de la revolución científica y de cualquier forma del desarrollo del conocimiento.

El libro de Pedro López, escrito en Cartagena de Indias, fue referente médico en todo el imperio donde no se ponía el sol y usado en varios continentes; tuvo vigencia hasta las primeras décadas del siglo XVIII, evidenciado en las 5 ediciones del libro que se imprimieron durante el siglo XVII. Otro aspecto para subrayar es que nuestro médico, a pesar de vivir en el lejano Caribe occidental, tuvo acceso a la bibliografía especializada, moderna y clásica del conocimiento médico y quirúrgico de la época, como lo demuestran sus más de 1200 citas y los casi 50 autores referenciados a lo largo de libro, además de un uso incipiente de la estadística en el registro de sus casos.

Por último, cerramos nuestras consideraciones con dos reflexiones más en torno al carácter científico y pedagógico de la obra. En primer lugar, cabe resaltar el Antidotario escrito en la parte final de la obra, que revela el ejercicio del conocimiento experimental con el uso innovador de plantas de ambos lados del Atlántico y las representaciones por medio de dibujos minuciosos de un laboratorio equipado con instrumentos de la época, que describe detalladamente en una relación de instrumentos ferrales y clínicos que creemos refuerza nuestra idea del profesionalismo de su trabajo. En segundo lugar, se debe señalar el rasgo de modernidad que implica que la obra sea escrita en lengua romance para transferir el conocimiento a los cirujanos romancistas que no tuvieron oportunidad de acceder a centros de estudios $y$ universidades. 


\section{PRATICA}

\section{Y T E
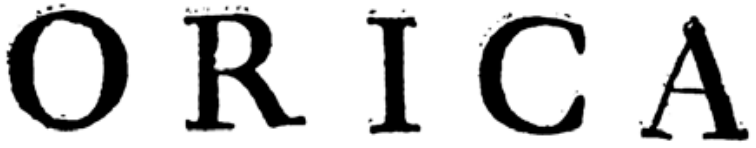 \\ DE LAS A POSTEMAS}

EN GENERAL; Y PARTICVLAR.

QVESTIONES, Y PRATICAS DE CIRVGIA; de heridas, llagas, y otras colas nuevas,

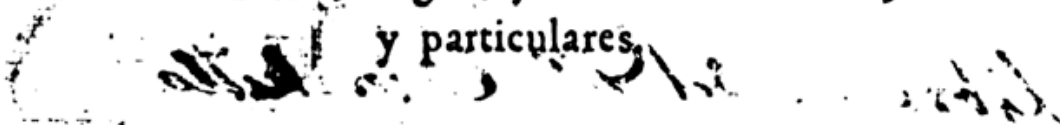
POR ELLICENCIADO PEDRO LOPEZ de Leon, Cirujano en la Ciudad de Cartagena de las Indias.

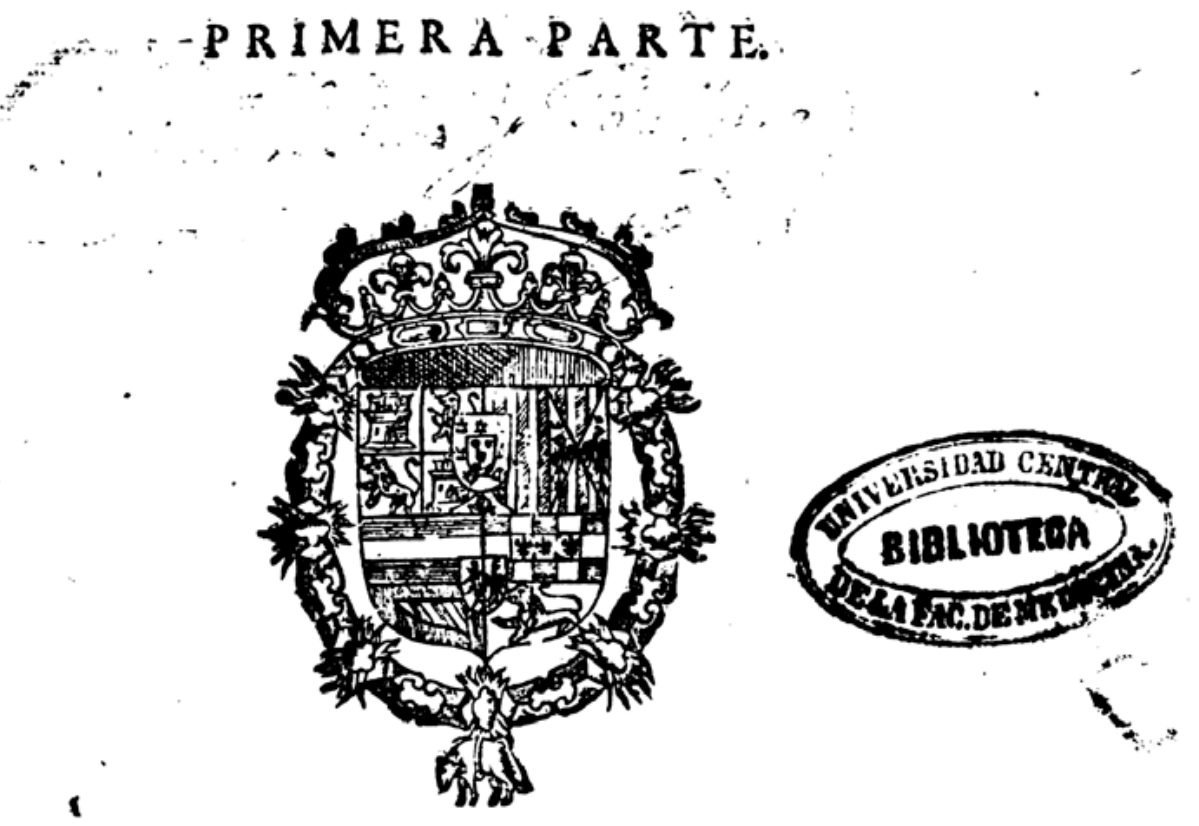

CON PRIVILEGIOO.

Impteffo en Sevilla, en la Oficina de Luys Eftupiñan. Año de 1628 . 


\section{NOTAS}

1 Esta investigación forma parte del Proyecto Connected Worlds: The Caribbean, Origin of Modern World". This project has received funding from the European Union's Horizon 2020 research and innovation programme under the Marie Sklodowska Curie grant agreement № 823846. Dirigido por Consuelo Naranjo Orovio desde el Instituto de Historia-CSIC.

2 La Inquisición se instaló en Cartagena de Indias en 1610.

3 Ejemplo de ello serían como resultado del diálogo de saberes entre los pueblos indígenas y los españoles, que dieron como resultado los códices Pomar en Perú y De la Cruz-Badiano, en Nueva España, publicados ambos afínales del siglo XVI.

4 Bartolomé Agüero Avisos de la cirugía contra la común opinión de publicada en 1584. Dionisio Daza Chacón Práctica y teórica de la cirugía en romance y en latín, 1582. Juan Fragoso, Cirugía universal, ahora nuevamente enmendada y añadida, 1601.

5 Deducimos de estos que los estudios y los doce primeros años de su carrera los desarrollo en Sevilla, pero la madurez profesional la alcanzó en Cartagena donde según nues-

\section{BIBLIOGRAFÍA}

Amaya, José Antonio (1986), Celestino Mutis y la expedición botánica, Madrid, Debate.

Agüero, Bartolomé (1584), Avisos de particulares de cirugía contra la común opinión. Sevilla, Casa de Francisco Pérez.

Bernal, Antonio Miguel (1992), La financiación de la Carrera de Indias (1492-1824): dinero y crédito en el comercio colonial español con América, Sevilla, Fundación el Monte.

Chaunu, Pierre (1977), Sevilla y América, siglos XVI y XVII, Sevilla, Universidad de Sevilla.

Dionisio Daza Chacón (1609), Practica y teórica de cirugía en romance y en latín, Valladolid, Casa de Ana Vélez, Biblioteca Virtual Miguel de Cervantes.

Fidalgo, Joaquín Francisco (2011), Derroteros y Cartografía de la expedición Fidalgo por el caribe neogranadino, 17921810, Bogotá, Ancora editores.

Fragoso, Juan (1601), Cirugía universal, aora nuevamente emendada, y añadida en esta sexta impressión, por el licenciado Juan Fragoso médico, y cirujano del Rey nuestro señor, y de sus Altezas. Y más otros quatro tratados. El primero es, una suma de proposiciones contra ciertos avisos de cirugía. El segundo, de las declaraciones acerca de diversas heridas, y muertes. El tercero, de los aforismos de Hippócrates tocantes a cirugía. El quarto, de la naturaleza, y calidades de los medicamentos simples, Madrid, editor Luis Sánchez.

Frías, Marcelo (1994), Tras el dorado vegetal, José Celestino Mutis y la Real expedición Botánica del Nuevo Reino de Granada 1783-1808, Sevilla, Diputación Provincial. tros cálculos vivió cerca de 30 años. En el libro aparece reiteradamente la frase a más de cuarenta años que practicó la medicina.

6 Pedro de Mercado, Recetas de espíritu para enfermos del cuerpo, Sevilla 1680.

7 Existen en total 5 ediciones del libro de Pedro López de León. El libro va a acompañado de la primera iconografía de instrumentos quirúrgicos en América.

8 Notas preliminares del libro.

9 Sobre el aspecto de la circulación de libros sugerimos el trabajo de Pedro Rueda, Las redes comerciales del libro en la colonia: peruleros y libreros en la Carrera de Indias 15801620, Anuario Estudios Americanos, 71, julio diciembre 2014, pp 447-478. Doi 10.3989/aeamer.2014.2.03

10 Reglas de cirugía: 368

11 Rama histórica de la ciencia que enlazaba la química y la medicina. Teniendo sus bases en la alquimia, la iatroquímica buscaba explicaciones químicas a los procesos patológicos y fisiológicos del cuerpo humano, y proporcionar tratamientos con sustancias químicas.

García, Antonio (2017), El mar de los deseos. El caribe Afroandaluz, historia y contrapunto, México, FCE.

García, Luis (1976), Historia social de la medicina en España, siglos XVI-XIII, Madrid, Akal.

Hernández, Helí (2011),"Juan Méndez Nieto el primer educador médico de Colombia", Revista Educación latinoamericana de UPTC, Vol. $13 \mathrm{~N}^{\circ} 17$, pp. 93-117.

Hernández, Emiliano (1960), La obra de López de León, Salamanca, Universidad de Salamanca.

Hernández, Guillermo (1982), Pensamiento científico y filosófico de José Celestino Mutis. Bogotá, Fondo cultural cafetero.

Humboldt, Alexander Von (1820). Le voyage aux régions equinoxiales du Nouveau Continent. Paris, Chez Maze.

Juan Jorge, Antonio de Ulloa (1748). Relación histórica del viaje a la América Meridional hecho de orden de SM para medir algunos grados de meridiano terrestre, Madrid, Antonio Marín editor.

Kamen, Henry (1997), Felipe de España, Madrid, Siglo XXI Editores.

Laín, Pedro (1977), Historia de la medicina, El saber médico en la antiguedad clásica, Barcelona, Salvat.

Leal, Abelardo (2018), José Celestino Mutis. Vida Obra y aportes científicos, económicos y políticos, Bogotá, Universidad Manuela Beltrán. 
López, José María (2007), Medicina e Historia natural en la sociedad española de los siglos XVI y XVII, Valencia, Universitat de Valencia.

López, José María (1991), El Códice Pomar (ca.1590) El interés de Felipe II por la Historia Natural y la Expedición Hernández a América, Valencia, Instituto de estudios documentales e históricos sobre la Ciencia, Universidad de Valencia-CSIC.

López, José María (1979), Ciencia y técnica en la Sociedad española de los siglos XVI y XVII, Barcelona, Editorial Labor.

Mantilla Luis Carlos; Díaz, Santiago (1944), Fray Diego García: su vida y su obra científica en la expedición Botánica, Bogotá, Academia colombiana de ciencias exactas, físicas y naturales.

Martínez, Gabriel (1986), Cartas de los Obispos de Cartagena durante el periodo Hispánico, 1534-1820, Medellín, Academia Colombiana de Historia Eclesiástica.

Mercado, Pedro (2006), Recetas de espíritu para enfermos del cuerpo, Sevilla 1680, Publicada en Programa de Apoyo al Desarrollo de Archivos Iberoamericanos-ADIA y la Universidad Nacional de Colombia.

Quevedo, Emilio (2008), Historia de la Medicina en Colombia. Tomo. I. Prácticas Médicas en conflicto, Bogotá, Tecnoquímicas- Norma.

Rodríguez, Sergio (2015), La Carrera de Indias. Las rutas, los hombres y las mercancías. Santander, La Huerta Grande.

Ronderos, Paula (2007), "El arte de boticario durante la primera mitad del siglo XVII en el Nuevo Reino de Granada", Revista Fronteras de la Historia del ICNH, 12, pp. 175-196.

Rueda, Pedro (2014), "Las redes comerciales del libro en la colonia: peruleros y libreros en la Carrera de Indias 15801620", Anuario Estudios Americanos, 71(2), pp. 447- 469.

Solano, Jairo (1998), Salud, Cultura y Sociedad en Cartagena de Indias, Barranquilla, Universidad del Atlántico.
Solano, Jairo (2007), "Juan Méndez Nieto y Pedro López de León: El arte de curar en la Cartagena del siglo XVII" (385443) en: Calvo, Haroldo y Meisel, Adolfo (ed.) Cartagena de Indias en el siglo XVII, Cartagena de Indias, Banco de la República.

Soto, Diana (2004), La reforma del plan de estudios del fiscal Moreno y Escandón 1774-1779, Bogotá, Universidad del Rosario.

Soto, Diana (2005), Mutis educador de la elite neogranadina, Tunja, Uptc.

Sotomayor Tribín, Hugo (1997), Guerras, enfermedades y médicos en Colombia. Bogotá, Escuela de Medicina Juan Corpas.

Sotomayor Tribín, Hugo (2009), “Cirujano licenciado Pedro López de León y su libro Práctica y teórica de las apostemas (siglo XVII)", Revista Repertorio, Vol 18, (1), pp. 53-64.

Sotomayor Tribín, Hugo (2003), "Sifilis y Pian: a propósito del morbo gálico en la obra de Pedro López de León. Cartagena de Indias a finales del siglo XVI y comienzos del siglo XVII." Temas Médicos. Vol XVI, Academia Nacional de Medicina, Bogotá 2003.

Tisnés, Roberto (1979), Don Pedro Fermín de Vargas, Bucaramanga, Academia de Historia de Santander.

Vidal, Antonino (2002), Cartagena de Indias y la región histórica del Caribe, 1580-1640. EEHH, Universidad de Sevilla.

Vidal, Antonino (2018), "Comercio y mercado regional en la gobernación de Santa Marta a finales del siglo XVIII: informe de José de Astigarraga 1789" (263-286), en; Von Grafestein Johanna (ed) . Entre lo legal, lo ilícito y lo clandestino. Prácticas comerciales y navegaciones en el Gran Caribe XVII-XIX, México, Instituto Mora.

Vila Enriqueta (1979), “Extranjeros en Cartagena de Indias 1593-1630". Hamburg: Jahrbuch für Geschichte Lateinamerikas JBLA, N. 16, pp. 147-184. 